\title{
Heterospecific mating and species recognition in the planktonic marine copepods Temora stylifera and T. longicornis
}

\author{
Erica Goetze $^{1,2, *}$, Thomas Kiørboe ${ }^{1}$ \\ ${ }^{1}$ National Institute of Aquatic Resources, Oceanography Section, Technical University of Denmark, Kavalergården 6, \\ 2920 Charlottenlund, Denmark \\ ${ }^{2}$ Present address: Department of Oceanography, University of Hawaii at Manoa, 1000 Pope Road, Honolulu, Hawaii 96822, USA
}

\begin{abstract}
Many planktonic copepods use diffusible pheromone or hydromechanical signals to remotely detect the presence of potential mates. To determine whether these mating signals also play a role in species recognition and mate choice, we observed and video recorded (3D) mate-finding and pursuit behaviors in heterospecific and conspecific mating crosses in a pair of congeneric, partially sympatric species (Temora stylifera and T. longicornis) in the laboratory. The species appear to have asymmetrical pre-mating isolation, with $T$. longicornis males readily pursuing $T$. stylifera females to mate contact and capture, but with little mate-finding activity observed in the reverse cross. Males of T. longicornis pursuing heterospecific females executed a number of behaviors known to facilitate successful pheromone trail following and mate capture in conspecific mating, including accelerated swimming in a 'signal-scanning' mode to recover a lost pheromone trail, reversal of the tracking direction in cases when the male initiated tracking in the incorrect direction, and accelerated swimming speeds when in the presence of a pheromone signal but prior to locating the trail. Detailed analyses of mate-tracking behavior in $T$. longicornis male $\times T$. stylifera female crosses gave no indication that males were aware they were pursuing heterospecific females prior to mate contact, indicating that diffusible pheromone and hydromechanical signals are not used, either singly or in combination, for species recognition in this mating pair. Heterospecific mating attempts among sympatric, congeneric copepods may commonly proceed to mate capture, and incur fitness costs to either or both mating partners.
\end{abstract}

KEY WORDS: Mate choice - Behavioral isolation · Mating asymmetry · Diffusible pheromone Hydromechanical signals

Resale or republication not permitted without written consent of the publisher

\section{INTRODUCTION}

Reproductive success in marine planktonic copepods requires appropriate mate choice in addition to mate encounter, suitable food availability and environmental conditions (e.g. Ianora et al. 2007, Titelman et al. 2007). One component of choosing an appropriate mating partner is discriminating heterospecific from conspecific potential mates. Temporal and spatial isolation between planktonic copepods is often incomplete due to broad biogeographic ranges and common sympatry with congenerics (e.g. Mullin 1969, Mullin \& Evans
1976, Frost 1989), and adults may encounter closely related heterospecifics as well as conspecifics as potential mating partners (Goetze 2008). Selective mate-choice behavior is therefore expected to be an important component to individual fitness. Mate choice has been little studied in marine planktonic copepods, but early observations of interspecific and intergeneric mating attempts suggest that 'mating errors' do occur and may be significant in the reproductive ecology of marine species (Katona 1973, Jacoby \& Youngbluth 1983).

For congeneric copepod species with incomplete temporal and spatial isolation, prezygotic mating barriers 
may occur through behavioral isolation during mate detection, pursuit or capture, mechanical isolation during mating, or post-mating prezygotic mechanisms such as gametic incompatibility or sperm competition (conspecific sperm precedence). Behavioral mechanisms provide the first line of defense in preventing heterospecific mating in the absence of temporal and spatial barriers, and are therefore the focus of the present study. Mate-finding in planktonic copepods falls into 3 broad strategies: (1) those in which the male detects and follows a discrete pheromone trail laid by the female ('trail-followers', Doall et al. 1998, Tsuda \& Miller 1998, Bagøien \& Kiørboe 2005a, Goetze 2008); (2) those in which the female produces a pheromone cloud or plume detected and penetrated by the male ('cloudpenetrators', Griffiths \& Frost 1976, Kiørboe et al. 2005, Kiørboe 2007); and (3) those in which both sexes generate and detect hydromechanical signals for mate location ('hoppers', Strickler 1998, Bagøien \& Kiørboe 2005b, Strickler \& Balázsi 2007). In mating modes (1) and (2), males are the active mate-seeking partner and use diffusible pheromone signals as the initial cue for mate detection. Diffusible pheromone signals persist far longer than hydromechanical signals in low Reynold's number environments (Yen et al. 1998), and can be used by males to detect and locate females at distances of 10 to >100 body lengths, long after any hydromechanical signal left in a female's wake has dissipated (van Duren et al. 1998, Yen et al. 1998). For both trail-followers and cloud-penetrators, therefore, mate-seeking males will initially detect a diffusible pheromone signal, followed by a hydromechanical signal when the male approaches within a few mm of the female. These pre-contact mating cues may be used either singly or in combination for mate recognition of conspecifics, and have been hypothesized to play an important role in reproductive isolation in copepods (Jacoby \& Youngbluth 1983, Buskey 1998, Lonsdale et al. 1998, Yen et al. 1998). Mechanical isolation due to morphological incompatibilities of sexual parts is also likely to be important in prezygotic isolation of some copepod groups (Fleminger 1967, Lee 1972, Blades 1977 , Blades-Eckelbarger 1991), and species recognition through contact chemoreception occurs in benthic harpacticoids (e.g. Frey et al. 1998, Ting \& Snell 2003, and references therein).

Temora stylifera and T. longicornis are congenerics that dominate epipelagic coastal and neritic waters of the North Atlantic and Mediterranean Sea (Fig. 1).
The species have overlapping, but largely separate, distributions. T. longicornis is a temperate species common to shelf waters of both northern European and American continents as well as in the North and Baltic Seas (e.g. CPR Team 2004, Razouls et al. 2005-2007 available at: http://copepodes.obs-banyuls.fr/en). $T$. stylifera occurs in abundance in subtropical and tropical coastal environments, including the Mediterranean Sea (e.g. Fleminger \& Hulsemann 1973, CPR Team 2004, Lindley \& Daykin 2005). A considerable northward shift has recently been observed in the northern boundary of the T. stylifera distribution along the European coast, and has been related to climate changes in the late 1980s (Villate et al. 1997, Lindley \& Daykin 2005). T. stylifera is now regularly present in moderately high abundance in the Bay of Biscay and coastal waters north to the British Isles during fall and early winter, perhaps due to advection from the south in the Warm Winter Poleward Current and increasing water temperate and stratification (e.g. Villate et al. 2004, Lindley \& Daykin 2005, Valdés et al. 2007, and references therein). This northward shift has increased the biogeographic overlap of the congeneric species pair, and may have increased heterospecific mate encounters between them. A portion of the population of both species is reproductively active during fall months in the western Mediterranean and North Seas (e.g. Halsband \& Hirche 2001, Halsband-Lenk et al. 2001, Di Capua \& Mazzocchi 2004). The reproductive ecology of these species is unknown in the region of biogeographic overlap.

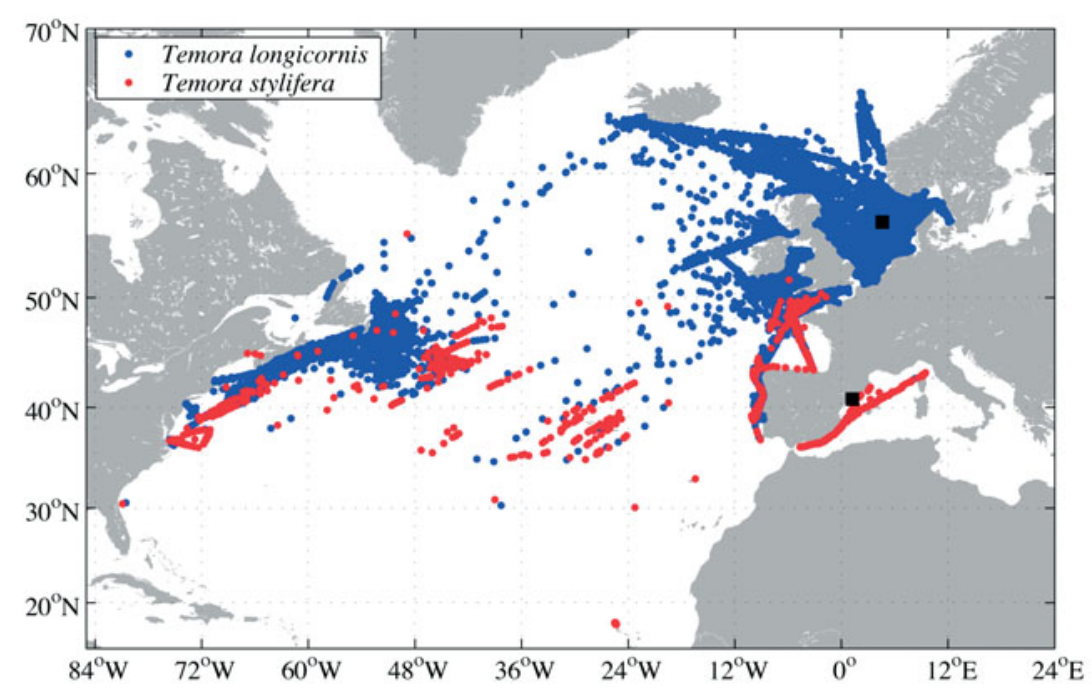

Fig. 1. Biogeographic distributions of Temora stylifera and T. longicornis in the North Atlantic and Mediterranean Sea, and origins of individuals used in mating experiments. $\mathbf{a}$ : collecting locations for T. longicornis (North Sea) and T. stylifera (Mediterranean Sea). Distribution records from the Ocean Biogeographic Information System (accessed 11 May 2007, www.iobis.org), derived primarily from the Continuous Plankton Recorder Survey 
Temora longicornis uses a 'trail-following' mate-finding strategy, with females leaving a discrete pheromone trail whose particular shape and dimensions depend on swimming behavior (Doall et al. 1998, Weissburg et al. 1998, Yen et al. 1998). Males exhibit behavioral flexibility during tracking, and use chemical gradients along and across the pheromone trail to detect and locate the female. Mate-finding and mating behaviors in $T$. stylifera have not been described previously.

We report simple observations of heterospecific and conspecific mate-finding behaviors in Temora stylifera and T. longicornis as part of a broader effort to identify mating signals involved in species recognition in planktonic copepods (Goetze 2008). Our primary goal was to determine whether pre-contact mating cues are used, either singly or in combination, as behavioral isolating mechanisms for sympatric congeneric copepod species.

\section{MATERIALS AND METHODS}

Collection and culture. Temora longicornis was established in continuous culture from specimens collected in the North Sea (Dogger Bank) in August 2005 (Fig. 1). Sea surface temperature and salinity during collection were $15.9^{\circ} \mathrm{C}$ and 34.6 , respectively. Individuals in culture were routinely fed mixed algal diets of Rhodomonas baltica, Heterocapsa triquetra, Thalassiosira weissflogii and Oxyhrris sp., as this diet was found to be sufficient for maintenance of the species through multiple generations in the laboratory (authors' pers. obs.). Copepods were reared under excess food conditions, and maintained at a salinity of 33 and temperature of 14 to $15^{\circ} \mathrm{C}$. These conditions that are within the annual range of sea surface temperature and salinity in the North Sea.

Temora stylifera was collected from coastal waters near Barcelona in the Mediterranean Sea in October and December 2005 (Fig. 1). Temperatures at $5 \mathrm{~m}$ depth during collection were 21.4 and $16.0^{\circ} \mathrm{C}$, and salinities were 36.1 and 37.7 in October and December, respectively. Sorted adults, copepodites and nauplii of T. stylifera were shipped or hand-carried to Denmark within a day of collection, and maintained at room temperature $\left(\sim 18^{\circ} \mathrm{C}\right)$ and a salinity of 36 or 38 . Individuals were fed mixed algal diets including Rhodomonas baltica, Heterocapsa triquetra, Oxyhrris sp., Prorocentrum minimum and Isochrysis galbana. The diet used for T. longicornis proved insufficient to support metamorphosis of $T$. stylifera nauplii to the first copepodite stage. We therefore augmented the $T$. stylifera diet with $P$. minimum and I. galbana, which are both highquality food for egg production and development of $T$. stylifera (Carotenuto et al. 2002, Ceballos \& Ianora 2003). When field-collected individuals reached maturity, they were used in mating experiments. T. stylifera was not successfully established in continuous culture.

Experimental individuals of Temora longicornis were obtained from the bulk culture by establishing a separate cohort by filtration of early stage nauplii through an 80 to $100 \mu \mathrm{m}$ mesh filter. This cohort was followed through development, and recently matured adults were used in mating experiments. The bulk culture was not synchronous, and the exact culture generation of experimental individuals was unknown. Given a development time of 3 to $4 \mathrm{wk}$ at this temperature, experimental individuals were likely in the 3rd to 6 th culture generation at the time of our experiments. Experimental individuals of both species were isolated by sex 1 to $4 \mathrm{~d}$ prior to experiments, to ensure that none had experienced recent matings. Only females not carrying spermatophores were included. Temora species are known to lack seminal receptacles and require frequent remating to produce fertile eggs (e.g. every 3 to 4 d; Ianora et al. 1989, Barthelemy et al. 1998, Corni et al. 2001). The isolation period was also used to acclimate individuals of each species to a common temperature of $18^{\circ} \mathrm{C}$ (room temperature) and salinity of 36 . Body sizes of the experimental individuals were not recorded, as they were kept alive in an attempt to establish a culture population ( $T$. sylifera). Mean adult female prosome length in $T$. stylifera collected at the same time and location as experimental individuals was $1.11 \pm 0.05(\mathrm{SD}) \mathrm{mm}(\mathrm{n}=23)$. Mean prosome length of adult female $T$. longicornis from the collection site was $1.0 \pm 0.04 \mathrm{~mm}(\mathrm{n}=23)$. Sizes of experimental females were likely smaller, as body size often declines in culture populations (T. Kiørboe pers. obs.). Average male antennule lengths in $T$. stylifera and T. longicornis were $1.45 \mathrm{~mm}( \pm 0.124 \mathrm{~mm}, \mathrm{n}=21)$ and $1.28 \mathrm{~mm}$ $( \pm 0.075 \mathrm{~mm}, \mathrm{n}=22)$, respectively. While the pre-histories of our experimental individuals differed (culture versus field-collected), we doubt that this was a confounding influence. Many T. stylifera individuals used in mating experiments were collected at sub-adult stages, and were not sexually active while in the field.

Mating experiments. We conducted both heterospecific and conspecific mating experiments. Heterospecific mating behaviors were observed in no-choice mating experiments with all specimens of each sex belonging to one species only. Seven heterospecific experiments were conducted with Temora stylifera females and $T$. longicornis males; 3 reverse cross experiments were conducted (Table 1). Three T. longicornis and $3 T$. stylifera conspecific experiments were used to verify the sexual activity of each population. We used individuals of the same generation as those used in heterospecific experiments. All experimental 


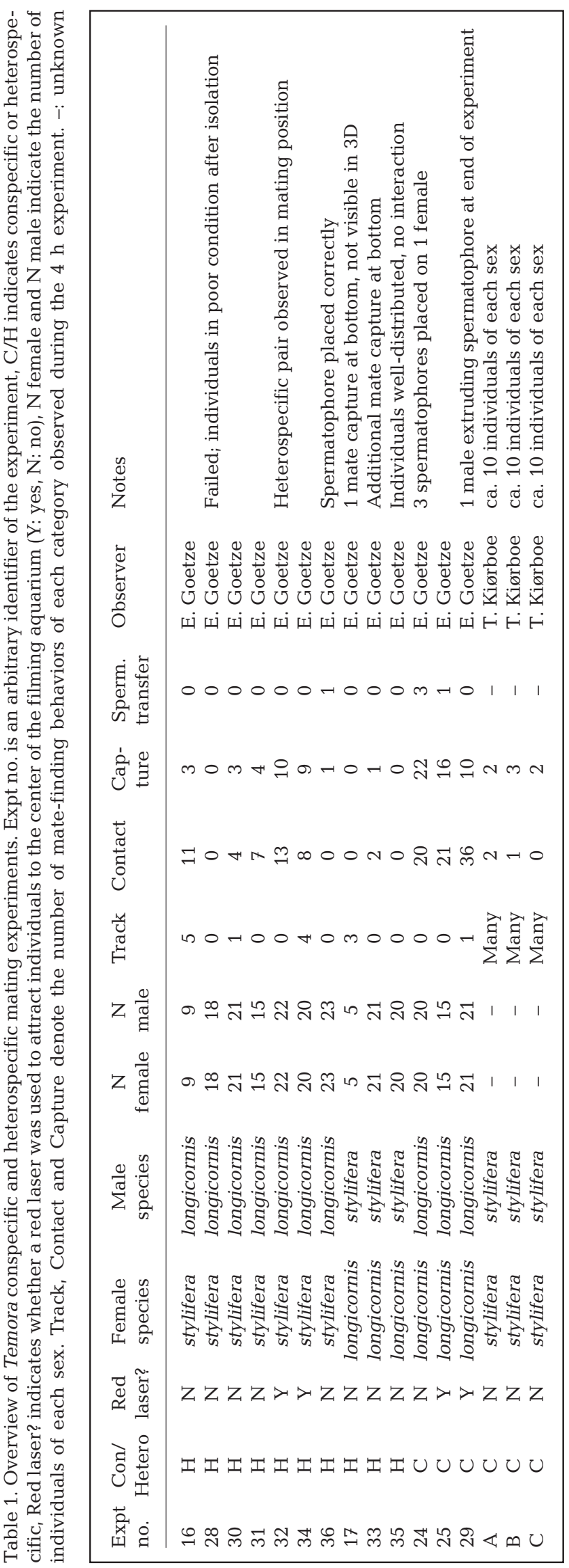

individuals were used only once. Mating experiments were conducted under saturating food conditions of Rhodomonas baltica and Prorocentrum minimum to ensure that appropriately sized food was available for each species. Heterospecific experiments were conducted at room temperature $\left(\sim 18^{\circ} \mathrm{C}\right)$ and a salinity of 36: conspecific $T$. longicornis experiments were run under rearing conditions (above). Densities ranged from 10000 to 46000 ind. $\mathrm{m}^{-3}$, within the range observed in field populations (e.g. Halsband-Lenk et al. 2004). Individuals were examined for the presence of spermatophores prior to and following treatments.

The experimental set-up for recording behavior in heterospecific and Temora longicornis conspecific experiments consisted of 2 synchronized monochrome CCD cameras with $50 \mathrm{~mm}$ lenses that viewed the $1 \mathrm{l}$ aquarium from orthogonal directions. Behavior was recorded continuously over a $4 \mathrm{~h}$ period. Individuals were filmed in the dark with collimated infrared lighting from behind (as described in Bagøien \& Kiørboe 2005a, Goetze 2008); they appeared as shadows in the video recordings. Each camera was connected to a video recorder (Panasonic AG-MD830, 25 frames $\mathrm{s}^{-1}$ ) via a time-stamp generator (Panasonic WJ 810, $1 / 100$ s), with a shared monitor for both views. Filming of a $1 \mathrm{~cm}$ ball at the start of each experiment was used for length calibrations. We tested a red laser as a possible attractant for these positively phototactic Temora species (Doall et al. 1998), as a way to minimize container wall effects on their behavior. The laser $(665 \mathrm{~nm}$, $1 \mathrm{~mm}$ wide light column) was mounted to provide a single, vertical column of light through the center of the aquarium. Early trials suggested that the laser acted as a mild attractant, facilitating complete 3D observations of mating behavior; it was included whenever possible in experiments (Table 1). Individuals were not concentrated directly within the column of light, but were present in the central portion of the water column. Conspecific experiments with T. stylifera were conducted on site in Barcelona (run by $\mathrm{T}$. Kiørboe) using a single CCD camera and a mirror placed in the diagonal of the aquarium to capture 3D views (as described in Kiørboe 2007) of mating behavior. The set-up was similar in all other respects to the 2-camera system.

Behavioral analysis. Mating behavioral sequences, or 'mating events', were categorized as (1) tracking, (2) contact or (3) capture events. Tracking events are sequences where the male detected the pheromone trail left by the female and initiated tracking behavior, but lost the trail prior to contact with the female. Contact events indicate sequences in which the male followed the pheromone trail to the point of mate contact, but failed to capture the female (typically the female hopped away at the capture lunge). Captures are events where 
the male successfully tracked a potential mate and succeeded in capturing her. Each mating sequence was included in only 1 category (i.e. 'contacts' did not precede 'captures'). Heterospecific mating interactions were readily distinguished from male-male interactions due to differences in body size between species.

Behavioral sequences selected for analysis were digitized using NIH ImageJ software (Image Processing and Analysis in Java available at: http://rsb.info. nih.gov), and 3D positions of the male and female were reconstructed using video recordings from both cameras. The time stamp on each video frame and vertical positions of the male in both views were used for synchronization of the 2 recordings. All heterospecific mate captures and contacts visible in 3D in their entirety were digitized, giving a total of 13 mating events. Complete 3D conspecific Temora longicornis mate captures and contacts were selected in chronological order, and a total of 24 mating interactions from 3 experiments were digitized. Six of 9 observed conspecific T. stylifera mating events were digitized, including all events visible in their entirety in 3D. All calculations of tracking behaviors were made on digitized sequences (Tables 2 , $3 \& 4$ ). In our calculations, the point at which males began tracking was defined by initiation of 'spinning' behavior, in which the male's position oscillated and he appeared to be rotating around the longitudinal body axis (Doall et al. 1998). During 'tracking', the male was directly following the pheromone trail. 'Pursuit' included the entire pre-contact mating sequence, which may have consisted of both 'tracking' and 'signalscanning' behaviors. Signal-scanning denotes a behavior in which the male accelerated above typical tracking speeds and searched a restricted volume to relocate a lost pheromone trail. Mann-Whitney $U, t$, and loglikelihood ratio $(G)$ tests were used with Bonferroni corrections to examine mean and frequency differences in male tracking behaviors between hetero- and conspecific mating events (Zar 1999).

\section{RESULTS}

\section{Conspecific mate-detection and pursuit}

In Temora stylifera, mate-finding proceeds through a 'trail-following' strategy in which the adult female leaves a discrete pheromone trail that mate-seeking males detect and follow until contact or capture of the female (Fig. 2, Table 2). Males of T. stylifera detected pheromone trails within $3 \mathrm{~mm}$ ( 3 body lengths) of the track-line, and accelerated to $25-30 \mathrm{~mm} \mathrm{~s}^{-1}$ during the mate chase (Table 2, Fig. 2). Males initiated tracking in both the correct and incorrect directions relative to the position of the female, and lost and recovered the

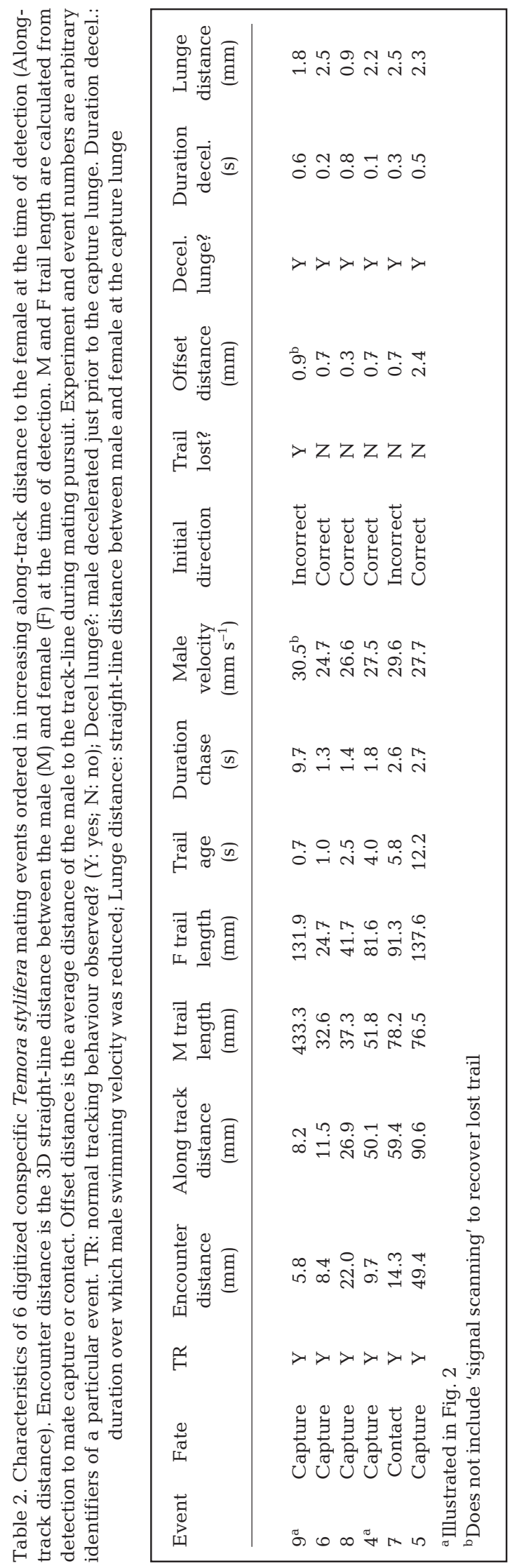




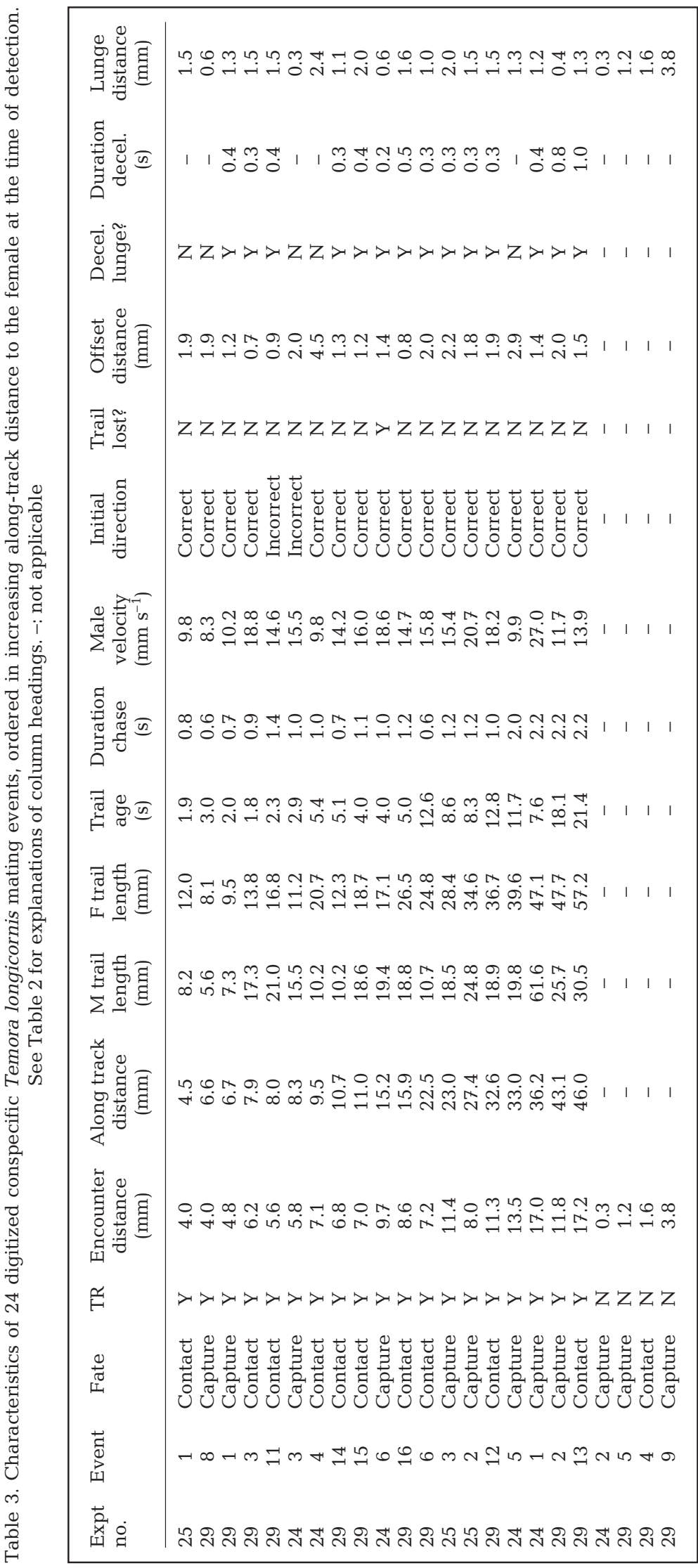

pheromone trail during the mate chase in some cases (Table 2, Fig. 2). Males also exhibited the 'spinning' behavior described for $T$. longicornis upon detection of the pheromone trail, and continued spinning throughout the mating pursuit (Doall et al. 1998). Fig. 2A,B illustrates a typical T. stylifera mating sequence in which the male detected the female pheromone trail (alongtrack distance to the female: $50.1 \mathrm{~mm}$ ), accelerated to an average swimming speed of $27.5 \mathrm{~mm} \mathrm{~s}^{-1}$ during tracking, and followed the pheromone trail to mate capture (Table 2, Event 4). In this case, the male shortened the tracking distance by not following the female on one helical swimming loop. The female crossed within $2.3 \mathrm{~mm}$ of her prior path, and the male detected the more recent trail. In the mating event described in Fig. 2C-G, the male initiated 'signal scanning' behavior upon loss of the pheromone trail, and accelerated above typical tracking speeds (to a mean of $48.5 \mathrm{~mm}$ $\mathrm{s}^{-1}$ ) while searching for the lost trail. The male relocated the trail after $7.7 \mathrm{~s}$ of searching, and successfully pursued the female to mate capture. The mate-finding behaviors described here for $T$. stylifera are broadly similar to those reported for T. longicornis (this study, Doall et al. 1998).

In the digitized Temora stylifera behavioral sequences, males pursued females over along-track distances up to $90.6 \mathrm{~mm}$ over a maximum of $9.7 \mathrm{~s}$ (Table 2). Males detected and responded to pheromone trails up to $12.2 \mathrm{~s}$ old, and tracked them at average offset distances of 0.4 to $2.4 \mathrm{~mm}$ from the track-line (Table 2). Female trail length ranged up to $137.6 \mathrm{~mm}$, and male pursuit distance ( $\mathrm{M}$ trail length) covered up to $433.3 \mathrm{~mm}$. Male pursuit distance was typically less than the female trail length, except in cases where the pheromone trail was lost or the male initiated tracking in the incorrect direction (e.g. Event 9, Table 2). Average male tracking velocity ranged from 26.6 to $30.5 \mathrm{~mm} \mathrm{~s}^{-1}$, somewhat higher than velocities in T. longicornis (Tables $2 \& 3$ ).

Conspecific Temora longicornis mating events in Table 3 were broadly similar to mate-finding behaviors observed in this species in prior studies (Doall et al. 1998, Weissburg et al. 1998). Males detected and followed pheromone trails up to $21.4 \mathrm{~s}$ old over total pursuit distances up to $61.6 \mathrm{~mm}$. 


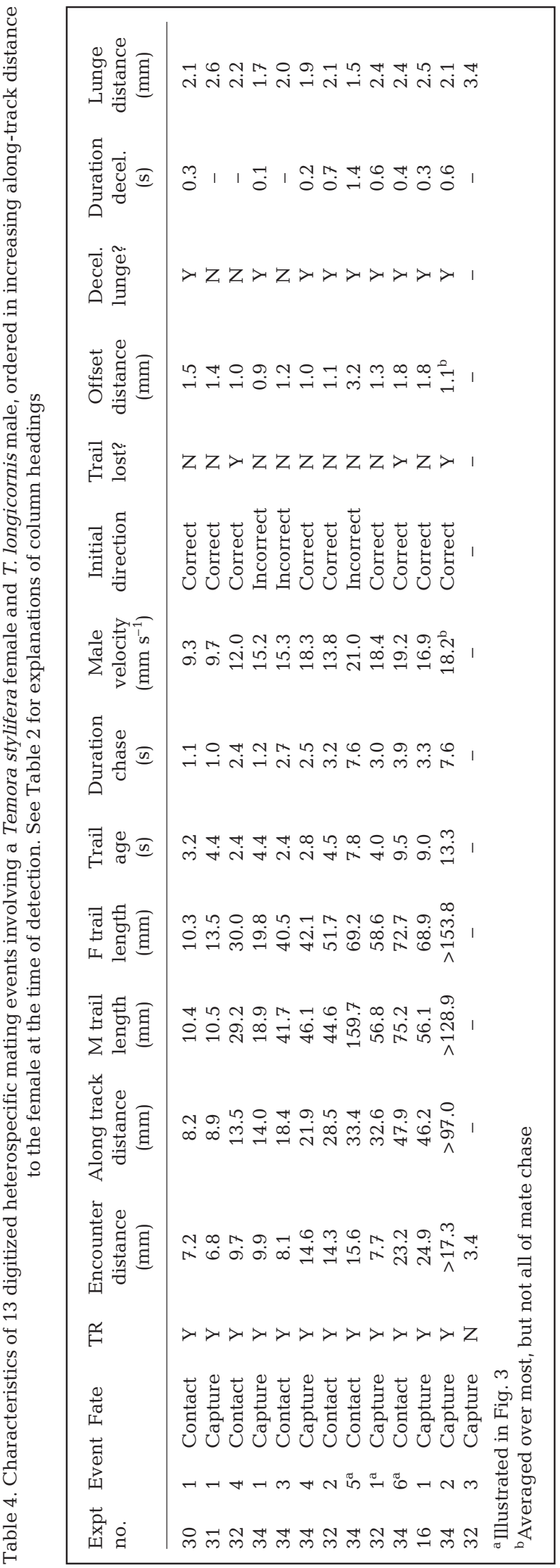

Males initiated tracking in the incorrect direction $11 \%$ of the time, and lost the trail in $5 \%$ of cases (Tables 3 \& $5)$. Average male velocity during tracking ranged from 8.3 to $27.0 \mathrm{~mm} \mathrm{~s}^{-1}$, and males followed pheromone trails at average offset distances of 0.7 to $4.5 \mathrm{~mm}$ from the track-line. Female swimming styles included both 'hovering' and 'cruising', with 'hovering' indicating slow-velocity swimming, typically with the body axis oriented vertically, and 'cruising' indicating highervelocity swimming (as described in Doall et al. 1998, Yen et al. 1998). Tracking behaviors of males pursuing 'hovering' and 'cruising' females appeared to differ, and events with low male velocities and high offset distances were those in which the male was tracking a 'hovering' female (e.g. Table 3, Expt 24, Event 4). In these events, the male was usually tracking below the original female position. Males decelerated immediately prior to the capture lunge in $74 \%$ of cases for durations of 0.2 to $1.0 \mathrm{~s}$ (Tables $3 \& 5$ ). Males lunged to capture the female at distances of 0.3 to $2.4 \mathrm{~mm}$ (Tables 3 \& 5). There were 4 cases of direct capture in which males lunged and captured females at distances of 0.3 to $3.8 \mathrm{~mm}$ (Table 3). We observed a total of 125 T. longicornis conspecific mating interactions in 3 experiments (Table 1).

\section{Heterospecific mating behavior in Temora stylifera and T. longicornis}

Heterospecific mating attempts between Temora stylifera females and T. longicornis males were largely indistinguishable from conspecific mating events of either species (Fig. 3). T. longicornis males detected and responded to $T$. stylifera pheromone trails that were up to $13.3 \mathrm{~s}$ old, and pursued heterospecific females for up to $160 \mathrm{~mm}$ total pursuit distance (Table 4). Males successfully captured females detected at up to $>97.0 \mathrm{~mm}$ away along the track-line, sometimes after initiating tracking in the incorrect direction ( $25 \%$ of cases) or losing and recovering the pheromone trail ( $25 \%$ of cases, Tables $4 \& 5)$. Males followed the track-line at average offset distances ranging from 0.9 to $3.2 \mathrm{~mm}$, swimming at velocities of 9.3 to $21.0 \mathrm{~mm} \mathrm{~s}^{-1}$ (Table 4). Table 4 includes events in which the male was pursuing either a 'hovering' or a 'cruising' female, and the difference was observed primarily in the velocity of the male during pursuit, which was generally $\leq 10 \mathrm{~mm} \mathrm{~s}^{-1}$ when chasing 'hovering' females. As observed in conspecific mating interactions, males also appeared to decelerate immediately prior to the lunge at mate capture (Doall et al. 1998). The duration of the deceleration prior to the mating lunge ranged from 0.1 to $1.4 \mathrm{~s}$ (Fig. 3, Table 5), and the distance from which the male lunged to capture the heterospecific female 


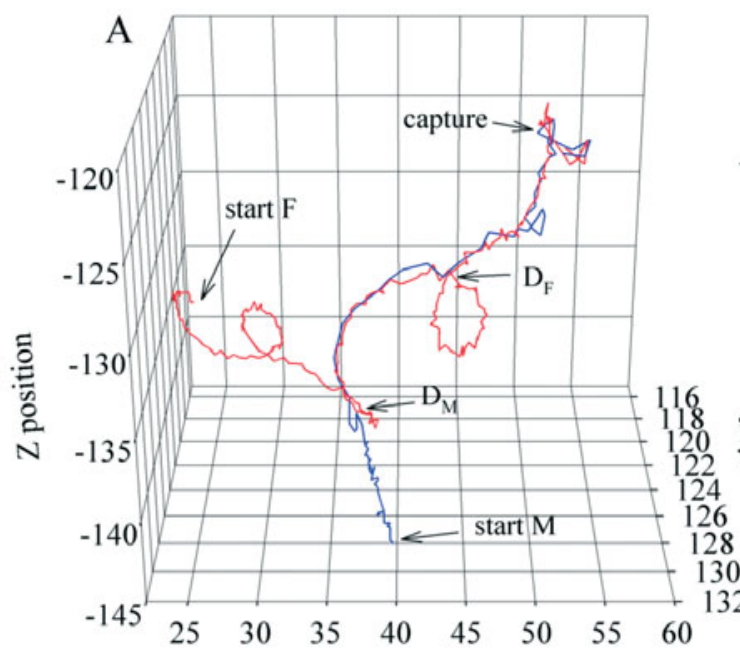

B
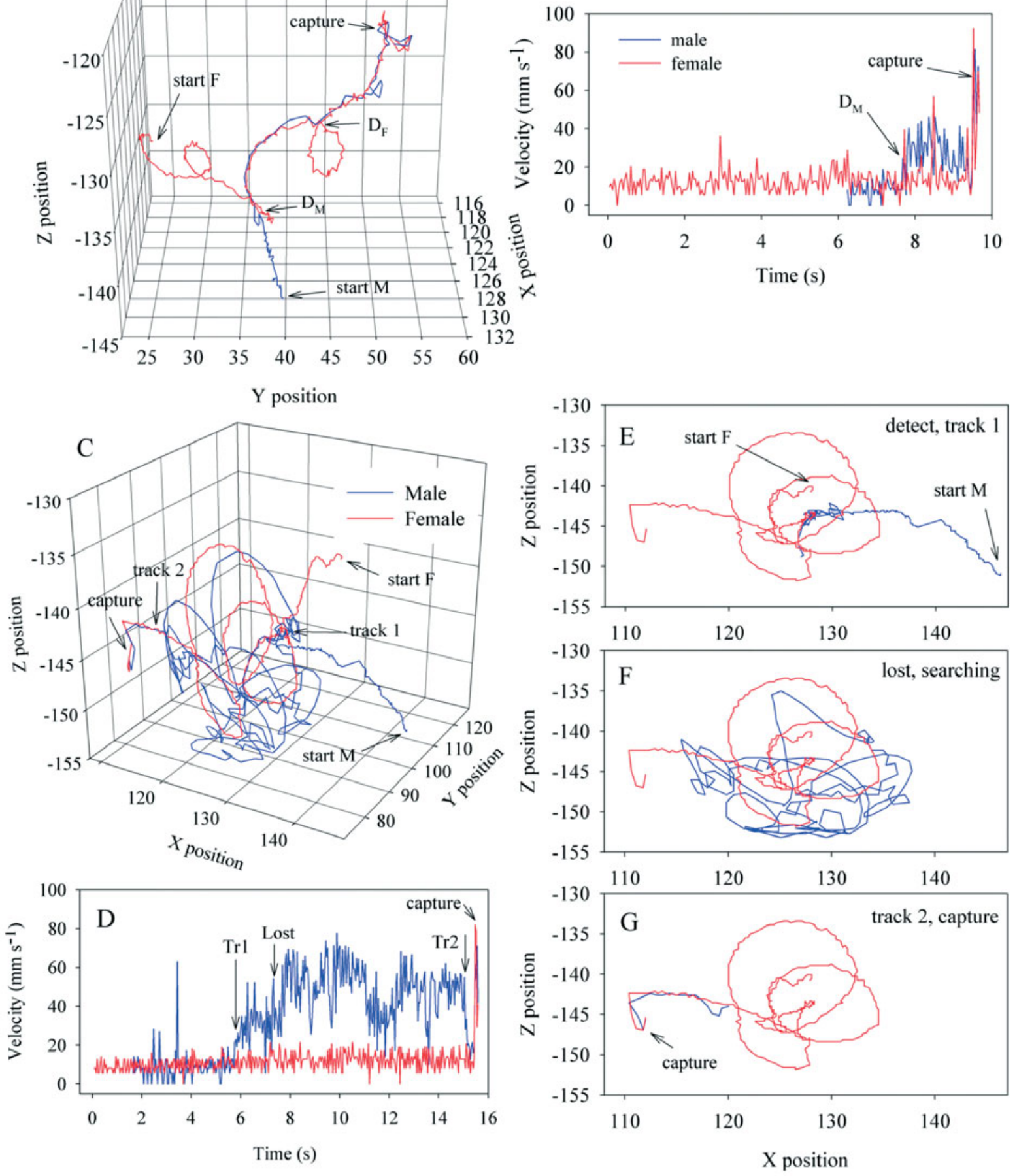

Fig. 2. Temora stylifera. Two examples of conspecific mating encounters. (A) \& (B) describe a typical mate capture (Table 2, Event 4); (C) $-(\mathrm{G})$ describe a mate capture during which the male initiated tracking in the incorrect direction, reversed direction, lost and recovered the pheromone trail, and finally captured the female after $15.5 \mathrm{~s}$ (Table 2, Event 9). (A) 3D swimming trajectories of male and female during the event. $\mathrm{D}_{\mathrm{M}}, \mathrm{D}_{\mathrm{F}}$ : positions of male and female at pheromone trail detection. (B) Swimming velocities of male and female before and during the event. (C) 3D swimming trajectories of male and female before and during the event. (D) Swimming velocities of male and female, with track 1 (Tr1), trail loss, track 2 (Tr2), and capture. Tr1 and Tr2 indicate detection of the pheromone trail and initiation of tracking behavior. (E-G) 2D swimming trajectories of the male and female before and during the event, with (E) initial trail detection and tracking (0 to $7.2 \mathrm{~s})$, (F) accelerated search for the pheromone trail ( 7.2 to $14.9 \mathrm{~s})$, and $(\mathrm{G})$ second trail detection, tracking, and mate capture (14.9 to $15.5 \mathrm{~s})$. See 'Results' for further details. Units for position are $\mathrm{mm}$, but are arbitrary relative to location within the aquarium 

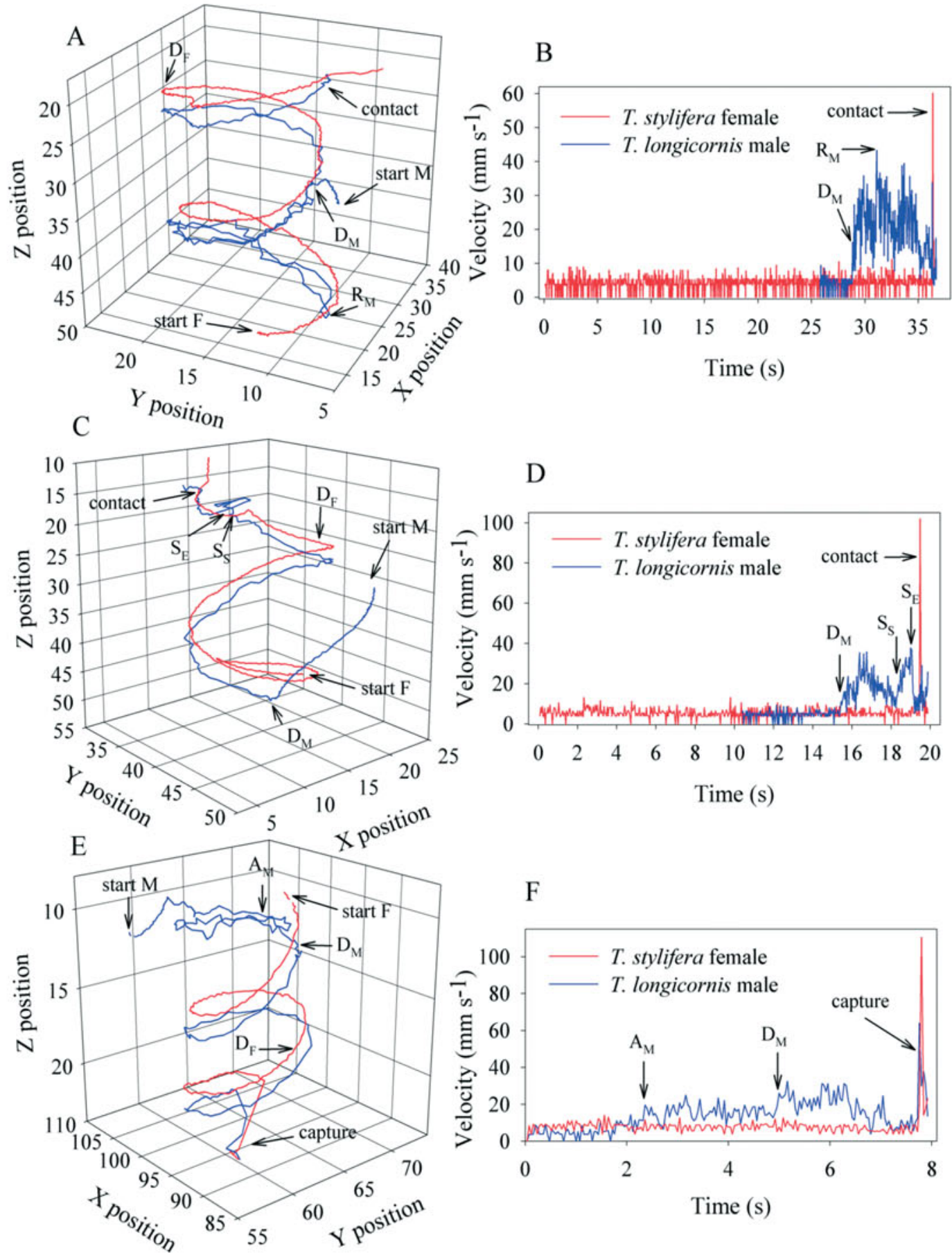

$\mathrm{F}$

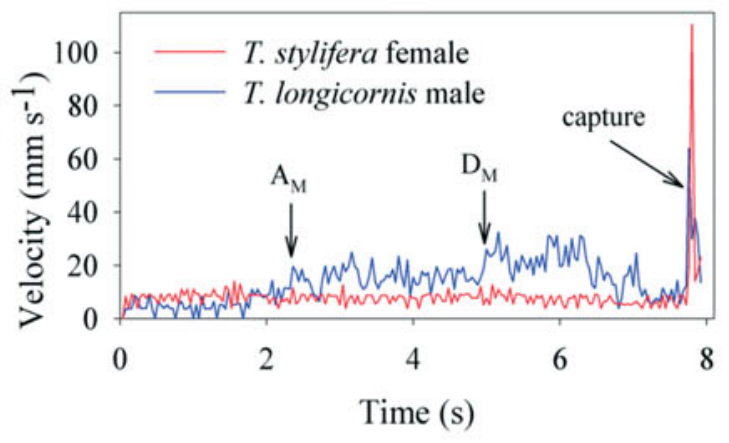

Fig. 3. Three examples of heterospecific mating encounters between a Temora stylifera female and T. longicornis male. (A) \& (B) describe a mate contact in which the male initiated tracking in the incorrect direction (Expt 34, Event 5, Table 1); (C) \& (D) describe a mate contact following loss and recovery of the pheromone trail (Expt 34, Event 6, Table 1); (E) \& (F) describe a mate capture with detection of the pheromone signal and male acceleration prior to tracking behavior (Expt 32, Event 1, Table 1). (A) Swimming trajectories of a T. longicornis male chasing and contacting a T. stylifera female. Male reverses direction at $R_{M}$. $D_{M}, D_{F}$ : locations of male and female when the pheromone trail was detected. (B) Swimming velocities of male and female before and during the mating event. (C) Swimming trajectories of a T. longicornis male chasing and contacting a T. stylifera female. $\mathrm{S}_{\mathrm{S}}$, $\mathrm{S}_{\mathrm{E}}$ : start and end of 'signal-scanning' behavior. (D) Swimming velocities of male and female before, during and after the mating event. (E) Swimming trajectories of T. longicornis male chasing and capturing a T. stylifera female. $\mathrm{A}_{\mathrm{M}}$ : acceleration of the male prior to locating the pheromone trail. (F) Swimming velocities of male and female before and during the mating event. See 'Results' for further details. Units for position are $\mathrm{mm}$, but are arbitrary relative to location within the aquarium 
ranged from 1.5 to $3.4 \mathrm{~mm}$ (Table 5). A total of 83 mating interactions were observed between this species pair (10 tracking events, 43 mate contacts, 30 mate captures; Table 1). One spermatophore was transferred between species in heterospecific experiments, and 1 additional hetereospecific pair was observed in the mating position at termination of the experiment.

We describe a few case examples below to illustrate how heterospecific mating interactions proceed between Temora stylifera females and T. longicornis males.

Case 1 (Fig. 3A,B, Table 4, Expt 34, Event 5): In this event, a Temora longicornis male detected the T. stylifera pheromone trail, initiated tracking in the incorrect direction, reversed, and then followed the helical track-line for an additional $5.3 \mathrm{~s}$ to mate contact. The male detected the female trail at a distance of $33.4 \mathrm{~mm}$ along the track-line, when the trail was $7.8 \mathrm{~s}$ old. Upon detection of the pheromone trail, the male began spinning and accelerated to an average speed of $21.0 \mathrm{~mm}$ $\mathrm{s}^{-1}$ from a background velocity of $3.8 \mathrm{~mm} \mathrm{~s}^{-1}$. The average offset distance to the pheromone trail during tracking was $3.2 \mathrm{~mm}$. As the male approached the female, he decelerated to $12.8 \mathrm{~mm} \mathrm{~s}^{-1}$ for $1.4 \mathrm{~s}$ prior to the capture lunge (Fig. 3B). The total pursuit distance was $159.7 \mathrm{~mm}$, and duration of the mate chase was $7.6 \mathrm{~s}$.

Case 2 (Fig. 3C,D, Table 4, Expt 34, Event 6): This event shows that Temora longicornis males may lose and successfully recover a $T$. stylifera pheromone trail during mate tracking. The $T$. longicornis male detected the pheromone trail, accelerated to an average speed of $19.2 \mathrm{~mm} \mathrm{~s}^{-1}$, and followed the trail for $51.3 \mathrm{~mm}$ until he lost the track-line at a point where the female executed a sharp turn. The male searched for the trail at higher velocity (25.3 $\mathrm{mm} \mathrm{s}^{-1}$, signal-scanning) for $0.6 \mathrm{~s}$ before relocating the trail and tracking the female to mate contact. The male also decelerated for $0.4 \mathrm{~s}$ as he approached the female, immediately prior to the capture lunge (Fig. 3D), but failed to capture the female.
The mating pursuit took place over $75.2 \mathrm{~mm}$ for a duration of $3.9 \mathrm{~s}$, with an average offset distance to the track-line of $1.8 \mathrm{~mm}$ during tracking.

Case 3 (Fig. 3E,F, Table 4, Expt 32, Event 1): In this event, a Temora longicornis male sensed the pheromone signal of a $T$. stylifera female and accelerated, but was unable to locate the trail immediately. The male searched for $2.4 \mathrm{~s}$ before locating the pheromone trail and tracking the female successfully to mate capture. Average male velocity during searching was $15.7 \mathrm{~mm} \mathrm{~s}^{-1}$, and was higher $\left(18.5 \mathrm{~mm} \mathrm{~s}^{-1}\right.$ ) during tracking. When tracking began, the female was $32.7 \mathrm{~mm}$ away along the track-line, and the trail was $4.0 \mathrm{~s}$ old. The male pursued the female over a total distance of $56.8 \mathrm{~mm}$, and was offset by an average of $1.3 \mathrm{~mm}$ from the pheromone trail during tracking. The male decelerated for $0.6 \mathrm{~s}$ immediately prior to the capture lunge (Fig. 3F).

In the reverse cross, between Temora stylifera males and $T$. longicornis females, little interaction was observed, although individuals were present at close proximity in high concentrations (10 to 44 ind. $\mathrm{l}^{-1}$ ) and with an observed high level of mating activity among T. stylifera males (male-male interactions indicate a readiness to mate). Only 6 mating attempts were observed (3 tracks, 2 contacts, 1 capture), none of which took place entirely free of the aquarium walls. No spermatophores were transferred between this species pair (Table 1).

\section{Male tracking behavior and species recognition}

Temora longicornis males did not have reduced ability to track heterospecific T. stylifera trails in comparison to conspecific mating encounters (Table 5). In this comparison, we were particularly interested in whether males in heterospecific mating events had higher rates

Table 5. Effectiveness of Temora longicornis male tracking behaviors in heterospecific (Temora stylifera female and T. longicornis male) and conspecific mating events (captures and contacts; Tables $2 \& 3$ ). Medians (ranges) listed for tracking parameters. Bold type: statistically significant $(\alpha=0.05$, Mann-Whitney $U$-test)

\begin{tabular}{|c|c|c|}
\hline Tracking parameter & Heterospecific & Conspecific \\
\hline Lost trail (\%) & 25 & 5 \\
\hline Incorrect initial tracking direction (\%) & 25 & 11 \\
\hline Male velocity during pursuit $\left(\mathrm{mm} \mathrm{s}^{-1}\right)$ & $16.11(9.31-21.01)$ & $14.66(8.27-27.04)$ \\
\hline Offset distance (mm) & $1.25(0.87-3.17)$ & $1.82(0.7-4.51)$ \\
\hline Duration of chase $(\mathrm{s})$ & $2.86(1.04-7.6)$ & $1(0.64-2.24)$ \\
\hline Trail age at detection (s) & $4.44(2.4-13.28)$ & $5.12(1.8-21.44)$ \\
\hline Length of pursued trail (mm) & $45.34(10.43-159.71)$ & $18.57(5.62-61.64)$ \\
\hline Along-track distance at time of detection (mm) & $25.22(8.19-96.5)$ & $15.22(4.48-46.01)$ \\
\hline Male deceleration at lunge $(\%)$ & 75 & 74 \\
\hline Duration of deceleration (s) & $0.36(0.12-1.4)$ & $0.34(0.16-1.04)$ \\
\hline Lunge distance (mm) & $2.1(1.5-3.4)$ & $1.3(0.3-2.4)$ \\
\hline
\end{tabular}


of tracking failure (trail loss, incorrect direction), lower tracking velocities, higher tracking offset distances, higher frequencies of deceleration prior to the capture lunge, or a longer duration of deceleration prior to capture, which would indicate either that males had greater difficulty pursuing heterospecific females or that they were able to detect that the female was heterospecific prior to contact. However, no significant differences were found between male tracking behaviors on heterospecific and conspecific pheromone trails in the frequency of trail loss, frequency of initiating tracking in the incorrect direction, male velocity during pursuit, distance to the pheromone trail during tracking, trail age at detection, along-track distance to the female at detection, frequency of male deceleration immediately prior to the capture lunge, or in the duration of deceleration (log-likelihood ratio, MannWhitney $U$ - and $t$-tests, $p>0.05$ in all cases, Table 5). In contrast, T. longicornis males pursued heterospecific females for longer tracking durations (Mann-Whitney $U$-test, $U=24.0, \mathrm{p}<0.005)$ and over longer total tracking distances (Mann-Whitney $U$-test, $U=43.0$, p < 0.005) than when they pursued conspecific females (Table 5). This suggests that (1) T. stylifera females produced longer pheromone trails than T. longicornis females, as expected given the difference in body size between species (Bagøien \& Kiørboe 2005a), and (2) that $T$. longicornis males were able to successfully pursue heterospecific females over their full pheromone trail length. T. longicornis males also lunged at mate capture from a greater distance to the female during heterospecific than conspecific mating attempts (MannWhitney $U$-test, $U=21.5, \mathrm{p}<0.005)$.

\section{DISCUSSION}

Observations on heterospecific mating behavior between Temora longicornis males and T. stylifera female indicate that diffusible pheromone and hydromechanical pre-contact mating signals are not used, either singly or in combination, for species recognition in this cross. T. longicornis males were readily able to detect and follow pheromone trails left by $T$. stylifera females to the point of mate contact and capture. Males also exhibited tracking behaviors that facilitate successful trail-following in normal conspecific matings, including accelerated swimming in a 'signal-scanning' mode to recover a lost pheromone trail, detection of along-track chemical gradients and reversal of tracking direction in cases when male tracking began in the incorrect direction, and accelerated swimming speeds when in the presence of a pheromone signal but prior to location of the trail (Fig. 3). These behaviors all indicate that trail detection and tracking in these hetero- specific mating interactions proceed largely the same way as observed in conspecific matings in these species (Doall et al. 1998, Weissburg et al. 1998, present study). Furthermore, there was no evidence that males were able to detect that females were heterospecific prior to mate contact as heterospecific and conspecific matings had similar failure rates (as trail loss and incorrect tracking direction), male tracking velocities, distances to the pheromone trail during tracking, trail ages at detection, along-track distances to the female at detection, and frequencies and durations of deceleration immediately prior to the mate capture lunge (Table 5). Results for the deceleration immediately prior to capture were of particular interest because males have access to information on both diffusible pheromone and hydromechanical signals produced by the female only during this final segment of the mate chase. Even if the diffusible pheromone signal were non species-specific and shared, for example, by multiple species within a genus or family, species recognition might occur through a combination of both chemical and hydromechanical signals immediately prior to contact. Distinct swimming behaviors generate unique hydromechanical disturbances (Yen \& Strickler 1996, van Duren et al. 1998, Yen et al. 1998, Visser 2001), and the detectable signal may vary between congeneric species. The fact that $T$. longicornis males engaged in heterospecific mating attempts did not prolong the deceleration step, decelerate in a higher proportion of mating interactions or abandon mate pursuit at this step in the mating sequence suggests that they do not evaluate the hydromechanical signal for species-specificity prior to lunge and capture. Hydromechanical signals, therefore, may be used only for orientation regarding the exact position of the female to ensure a successful catch (as suggested by Yen et al. 1998).

Preliminary observations on the reverse cross (Temora stylifera male $\times T$. longicornis female) showed little sexual activity, and it appears that pre-mating isolation may be asymmetrical between these species. However, given the absence of data on the frequency of mating behaviors in carefully controlled experiments, this inference is preliminary and requires corroboration. Lower sexual activity in this mating cross may result from at least 3 proximate causes: (1) reduced encounter rates between these mating partners due to the shorter pheromone trails laid by $T$. longicornis females (an 'encounter isolation' model); (2) reduced signal size in this mating pair due to bodysize related differences in signal strength and sensor sensitivity (a 'signal strength isolation' model); or (3) male $T$. stylifera detect the diffusible pheromone trail produced by $T$. longicornis females as heterospecific, and usually fail to initiate tracking behavior (or initiate tracking with lower probability: a 'behavioral isolation' 
model). We can examine whether simple encounter limitations are likely to place constraints on mating interactions in this heterospecific pair by calculating the male mate-search volume rates of $T$. stylifera and $T$. longicornis males searching for heterospecific potential mates. Kiørboe \& Bagøien (2005) described an encounter model for a cruising male pursuing a discrete pheromone trail (as in Temora species):

$$
\beta_{\text {trail,cruiser }}=2 L u_{2 \mathrm{D}}\left(\sqrt{\frac{D_{\mathrm{p}} L}{V}}+S\right)
$$

where $L$ is the length of the pheromone trail, $u_{2 \mathrm{D}}$ is the 2D swimming velocity of the male, $D_{\mathrm{p}}$ is the diffusion coefficient of the pheromone (taken here as $10^{-5} \mathrm{~cm}^{2} \mathrm{~s}^{-1}$ ), $\mathrm{v}$ is the 3D swimming velocity of the female, and $S$ is the sensory reach of the male (antennule length). Using swimming velocities for both species reported in Kiørboe (2008) and maximal pheromone trail lengths from this study, we find that $T$. stylifera and $T$. longicornis males are able to search 92 and $67 \mathrm{ld}^{-1}$, respectively, for heterospecific females. Therefore, although $T$. longicornis females produce shorter pheromone trails, reducing the spatial extent over which they are detectable by males, higher male velocities in $T$. stylifera should apparently counteract this effect in heterospecific mating crosses. In sum, males of each species have approximately equivalent probabilities of encounters with heterospecific pheromone trails, and simple encounter limitations are unlikely to explain differences in mating activity between the 2 heterospecific crosses.

Differences in signal strength and detection thresholds between species may also influence heterospecific mating interactions, resulting in asymmetrical premating isolation. Pheromone and hydromechanical signal production varies as a function of body size, with larger-bodied species able to generate greater hydromechanical disturbances and longer pheromone trails (e.g. Visser 2001, Bagøien \& Kiørboe 2005a). Smallerbodied species may also have lower detection thresholds, due to less self-generated noise. Mating interactions involving the larger-bodied female Temora stylifera and smaller-bodied male, T. longicornis could therefore result in the perception of larger signal size than in reverse cross mating interactions. Our observations are consistent with this signal strength isolation model. T. longicornis males chased T. stylifera females over longer total pursuit distances and for longer pursuit durations than conspecific females (Table 5), indicating that the larger-bodied females likely produce longer pheromone trails. Observations on conspecific mating in $T$. stylifera also suggest a longer pheromone trail length. Even with relatively few events analyzed, we find along-track distances to the female up to $90 \mathrm{~mm}$ for $T$. stylifera (Table 2), twice as long as the maximum observed along-track distance for $T$. longi- cornis in this study (Table 4). The pheromone trail length of $T$. stylifera may be underestimated here, because long tracking events are typically rare (Bagøien \& Kiørboe 2005a). Males and females of T. stylifera swim faster than T. longicornis (means: 7.2 versus $3.0 \mathrm{~mm} \mathrm{~s}^{-1}$ male, 3.3 versus $1.4 \mathrm{~mm} \mathrm{~s}^{-1}$ female, respectively, Kiørboe 2008), and are expected to generate greater hydromechanical disturbances. T. longicornis males also executed the lunge at mate capture from a greater distance to the female in heterospecific than in conspecific mating attempts (Table 5), which is consistent with the expectation that $T$. stylifera females produce a stronger hydromechanical signal that is detectable by males from greater distance. Combined, these observations suggest that the perception of signal size may be greater in the $T$. stylifera female $\times T$. longicornis male cross, resulting in higher heterospecific mating activity in this pair than in the reverse heterospecific cross ( $T$. longicornis female $\times T$. stylifera male). Asymmetrical pre-mating isolation may result from species differences in signal generation and sensitivity.

A third explanation for asymmetrical pre-mating isolation in these Temora congeners is that $T$. stylifera males may have some ability to discriminate heterospecific from conspecific females at early stages of mate-finding, and be choosy in their pursuit of potential mates (unlike T. longicornis, i.e. a behavioral isolation model). With our current observations, we cannot distinguish signal strength from behavioral isolation models. Asymmetrical mating isolation is not uncommon in congeneric insect species (e.g. Kaneshiro \& Giddings 1987, Coyne et al. 1994, Shaw \& Lugo 2001).

Diffusible pheromone and hydromechanical precontact mating signals were found to be non-speciesspecific in both the Temora longicornis male $\times$ T. stylifera female cross and a congeneric Centropages species pair (Goetze 2008). Mate-seeking males are apparently unaware of the species identity of the female prior to contact, and heterospecific mating attempts readily proceed to mate capture. Low rates of spermatophore placement in heterospecific Centropages crosses indicate that reproductive isolation is present, though not complete, and that species recognition occurred at mate capture (Goetze 2008). Spermatophore placement rates were uniformly low in the present study, and do not provide reliable information on reproductive isolating barriers. Observations of inter-generic mating attempts between $T$. longicornis males and $C$. typicus females further suggest that the diffusible pheromone signal is non-specific in many cases (Goetze 2008). Heterospecific mating attempts may be a common feature of the reproductive ecology of sympatric congeneric species, and may affect individual fitness. 
Acknowledgements. This study was funded by the Danish Natural Research Council (Grant 21-05-0024) and a Marie Curie Incoming International Fellowship (to E.G.). J. Melbye assisted with copepod and algal culturing, and M. Dünweber digitized some of the mating sequences. E. Bagiøen provided early instruction on the experimental set-up. We thank 3 anonymous reviewers for insightful comments on the manuscript.

\section{LITERATURE CITED}

Bagøien E, Kiørboe T (2005a) Blind dating - mate finding in planktonic copepods. I. Tracking the pheromone trail of Centropages typicus. Mar Ecol Prog Ser 300:105-115

Bagøien E, Kiørboe T (2005b) Blind dating - mate finding in planktonic copepods. III. Hydromechanical communication in Acartia tonsa. Mar Ecol Prog Ser 300:129-133

Barthelemy RM, Cuoc C, Defaye D, Brunet M, Mazza J (1998) Female genital structures in several families of Centropagoidea (Copepoda: Calanoida). Phil Trans R Soc Lond B Biol Sci 353:721-736

Blades PI (1977) Mating behavior of Centropages typicus (Copepoda: Calanoida). Mar Biol 40:57-64

Blades-Eckelbarger PI (1991) Functional morphology of spermatophores and sperm transfer in calanoid copepods. In: Bauer RT, Martin JW (eds) Crustacean sexual biology. Columbia University Press, New York, p 246-270

Buskey EJ (1998) Components of mating behavior in planktonic copepods. J Mar Syst 15:13-21

> Carotenuto Y, Ianora A, Buttino I, Romano G, Mitalto A (2002) Is postembryonic development in the copepod Temora stylifera negatively affected by diatom diets? J Exp Mar Biol Ecol 276:49-66

Ceballos S, Ianora A (2003) Different diatoms induce contrasting effects on the reproductive success of the copepod Temora stylifera. J Exp Mar Biol Ecol 294:189-202

> Corni MG, Vigoni V, Scanabissi F (2001) Some ultrastructural features of the reproductive morphology and spermatophore placement of Temora stylifera Dana, 1849 (Copepoda, Calanoida). Crustaceana 74:79-90

> Coyne JA, Crittenden AP, Mah K (1994) Genetics of a pheromonal difference contributing to reproductive isolation in Drosophila. Science 265:1461-1464

CPR (Continuous Plankton Recorder Survey) Team (2004) Continuous plankton records: plankton atlas of the North Atlantic Ocean (1958-1999). II. Biogeographical charts. Mar Ecol Prog Ser (Suppl):11-75. Available at: www.intres.com/ articles/CPRatlas/CPRp011.pdf

Di Capua I, Mazzocchi MG (2004) Population structure of the copepods Centropages typicus and Temora stylifera in different environmental conditions. ICES J Mar Sci 61: 632-644

Doall MH, Colin SP, Strickler JR, Yen J (1998) Locating a mate in 3D: the case of Temora longicornis. Phil Trans R Soc Lond B Biol Sci 353:681-689

Fleminger A (1967) Taxonomy, distribution, and polymorphism in the Labidocera jollae group with remarks on evolution within the group (Copepoda: Calanoida). Proc US Natl Mus 120:1-61

Fleminger A, Hulsemann K (1973) Relationship of Indian Ocean epiplanktonic calanoids of the world ocean. In: Zeitzschel B (ed) The biology of the Indian Ocean. Springer-Verlag, New York, p 339-347

> Frey MA, Lonsdale DJ, Snell TW (1998) The influence of contact chemical signals on mate recognition in a harpacticoid copepod. Phil Trans R Soc Lond B Biol Sci 353: $745-751$
Frost BW (1989) A taxonomy of the marine calanoid copepod genus Pseudocalanus. Can J Zool 67:525-551

Goetze E (2008) Heterospecific mating and partial prezygotic reproductive isolation in the planktonic marine copepods Centropages typicus and Centropages hamatus. Limnol Oceanogr 53:433-445

Griffiths AM, Frost BW (1976) Chemical communication in the marine planktonic copepods Calanus pacificus and Pseudocalanus sp. 1. Crustaceana 30:1-8

Halsband C, Hirche HJ (2001) Reproductive cycles of dominant calanoid copepods in the North Sea. Mar Ecol Prog Ser 209:219-229

Halsband-Lenk C, Nival S, Carlotti F, Hirche H (2001) Seasonal cycles of egg production of two planktonic copepods, Centropages typicus and Temora stylifera, in the north-western Mediterranean Sea. J Plankton Res 23: 597-609

Halsband-Lenk C, Carlotti F, Greve W (2004) Life-history strategies of calanoid congeners under two different climate regimes: a comparison. ICES J Mar Sci 61:709-720

Ianora A, di Carlo S, Mascellaro P (1989) Reproductive biology of the planktonic copepod Temora stylifera. Mar Biol 101:187-194

Ianora A, Miralto A, Halsband-Lenk C (2007) Reproduction, hatching success, and early naupliar survival in Centropages typicus. Prog Oceanogr 72:195-213

> Jacoby CA, Youngbluth MJ (1983) Mating behavior in three species of Pseudodiaptomus (Copepoda: Calanoida). Mar Biol 76:77-86

Kaneshiro KY, Giddings LV (1987) The significance of asymmetrical sexual isolation and the formation of new species. Evol Biol 21:29-43

Katona SK (1973) Evidence for sex pheromones in planktonic copepods. Limnol Oceanogr 18:574-583

Kiørboe T (2007) Mate finding, mating, and population dynamics in a planktonic copepod Oithona davisae: there are too few males. Limnol Oceanogr 52:1511-1522

> Kiørboe T (2008) Optimal swimming strategies in matesearching pelagic copepods. Oecologia 155:179-192

Kiørboe T, Bagøien E (2005) Motility patterns and mate encounter rates in planktonic copepods. Limnol Oceanogr 50:1999-2007

> Kiørboe T, Bagøien E, Thygesen UH (2005) Blind dating mate finding in planktonic copepods. II. The pheromone cloud of Pseudocalanus elongatus. Mar Ecol Prog Ser 300: $117-128$

Lee CM (1972) Structure and function of the spermatophore and its coupling device in the Centropagidae (Copepoda, Calanoida). Bull Mar Ecol 8:1-20

Lindley JA, Daykin S (2005) Variations in the distributions of Centropages chierchiae and Temora stylifera (Copepoda: Calanoida) in the northeastern Atlantic Ocean and western European shelf waters. ICES J Mar Sci 62:869-877

Lonsdale DJ, Frey MA, Snell TW (1998) The role of chemical signals in copepod reproduction. J Mar Syst 15:1-12

Mullin MM (1969) Distribution, morphometry and seasonal biology of the planktonic copepods Calanus tenuicornis and Calanus lighti in the Pacific Ocean. Pac Sci 23: 438-446

Mullin MM, Evans PM (1976) Distribution, morphometry and seasonal biology of the planktonic copepods Neocalanus robustior and Neocalanus gracilis in the Pacific Ocean. Pac Sci 30:119-130

> Shaw KL, Lugo E (2001) Mating asymmetry and the direction of evolution in the Hawaiian cricket genus Laupala. Mol Ecol 10:751-759

> Strickler JR (1998) Observing free-swimming copepods mating. Phil Trans R Soc Lond B Biol Sci 353:671-680 
Strickler JR, Balászi G (2007) Planktonic copepods reacting selectively to hydrodynamic disturbances. Phil Trans R Soc Lond B Biol Sci 362:1947-1958

Ting JH, Snell TW (2003) Purification and sequencing of a mate-recognition protein from the copepod Tigriopus japonicus. Mar Biol 143:1-8

Titelman J, Varpe Ø, Eliassen S, Fiksen Ø (2007) Copepod mating: chance or choice? J Plankton Res 29:1023-1030

Tsuda A, Miller CB (1998) Mate-finding behavior in Calanus marshallae. Phil Trans R Soc Lond B Biol Sci 353:713-720

Valdés L, López-Urrutia A, Cabal J, Alvarez-Ossorio M and others (2007) A decade of sampling in the Bay of Biscay: what are the zooplankton time series telling us? Prog Oceanogr 74:98-114

van Duren LA, Stamhuis EJ, Videler JJ (1998) Reading the copepod personal ads: increasing encounter probability with hydromechanical signals. Phil Trans R Soc Lond B Biol Sci 353:691-700

Villate F, Moral M, Valencia V (1997) Mesozooplankton community indicates climate changes in a shelf area of the

Editorial responsibility: William Peterson,

Newport, Oregon, USA inner Bay of Biscay throughout 1988 to 1990. J Plankton Res 19:1617-1636

Villate F, Uriarte I, Irigoien X, Beaugrand G, Cotano U (2004) Zooplankton communities. In: Borja Á, Collins M (eds) Oceanography and marine environment of the Basque Country. Elsevier Oceanogr Ser 70:395-423

Visser AW (2001) Hydromechanical signals in the plankton. Mar Ecol Prog Ser 222:1-24

> Weissburg MJ, Doall MH, Yen J (1998) Following the invisible trail: kinematic analysis of mate-tracking in the copepod Temora longicornis. Phil Trans R Soc Lond B Biol Sci 353:701-712

Yen J, Strickler JR (1996) Advertisement and concealment in the plankton: what makes a copepod hydrodynamically conspicuous? Invertebr Biol 115:191-205

Yen J, Weissburg MJ, Doall MH (1998) The fluid physics of signal perception by mate-tracking copepods. Phil Trans R Soc Lond B Biol Sci 353:787-804

Zar JH (1999) Biostatistical analysis. Prentice Hall, Englewood Cliffs, NJ

Submitted: December 5, 2007; Accepted: June 17, 2008

Proofs received from author(s): October 8, 2008 